\title{
Existence results for fractional differential inclusions arising from real estate asset securitization and HIV models
}

\author{
Bashir Ahmad ${ }^{1 *}$ and Sotiris K Ntouyas ${ }^{2}$
}

\section{"Correspondence:}

bashir_qau@yahoo.com

'Department of Mathematics,

Faculty of Science, King Abdulaziz

University, P.O. Box 80203, Jeddah,

21589, Saudi Arabia

Full list of author information is

available at the end of the article

\begin{abstract}
This paper studies a new class of boundary value problems of nonlinear differential inclusions with Riemann-Liouville integral boundary conditions arising from real estate asset securitization and HIV models. Some new existence results are obtained by using standard fixed point theorems when the right-hand side of the inclusion has convex as well as non-convex values. Some illustrative examples are also discussed.
\end{abstract} MSC: $34 \mathrm{~A} 60 ; 34 \mathrm{~A} 08$

Keywords: fractional differential inclusions; integral boundary conditions; fixed point theorems

\section{Introduction}

In this paper, we study the following boundary value problem:

$$
\left\{\begin{array}{l}
-D^{\alpha} x(t) \in F\left(t, x(t),-D^{\beta} x(t)\right), \quad 0<t<1,2<\alpha \leq 3, \\
D^{\beta} x(0)=D^{\beta+1} x(0)=0, \quad D^{\beta} x(1)=\int_{0}^{1} D^{\beta} x(s) d A(s),
\end{array}\right.
$$

where $D^{\alpha}$ is the standard Riemann-Liouville fractional derivative of order $2<\alpha \leq 3,0<$ $\beta<1, \alpha-\beta>2, F:[0,1] \times \mathbb{R} \times \mathbb{R} \rightarrow \mathcal{P}(\mathbb{R})$ is a multivalued map, $\mathcal{P}(\mathbb{R})$ is the family of all subsets of $\mathbb{R}, \int_{0}^{1} D^{\beta} x(s) d A(s)$ denotes the Riemann-Stieltjes integral, and $A$ is a function of bounded variation.

The subject of fractional differential equations has evolved as an interesting and important field of research in view of its numerous applications in physics, mechanics, chemistry, engineering (like traffic, transportation, logistics etc.), and so forth [1-3]. The tools of fractional calculus have played a key role in improving the mathematical modeling of many real world processes based on classical calculus. The nonlocal characteristic of a fractional order differential operator distinguishes it from a classical integer-order differential operator. In fact, differential equations of arbitrary order are capable of describing memory and hereditary properties of some important and useful materials and processes. For some recent development on the topic, see [4-19] and the references cited therein.

On the other hand, the nonlocal condition given by a Riemann-Stieltjes integral is due to Webb and Infante in [20] and gives a unified approach to many BVPs. Since $\int_{0}^{1} D^{\beta} x(s) d A(s)$ covers a multipoint BVP and an integral BVP as special cases, the fractional differential 
equations with the Riemann-Stieltjes integral condition were extensively studied by many authors, see [21, 22].

The problems of type (1.1) are referred to as semipositone problems that arise naturally in chemical reactor theory in the literature [23]. Recently, by SWOT analysis method, one has found that many mathematical models arising from real estate asset securitization can be interpreted by fractional-order differential or difference equations under suitable initial conditions or boundary conditions. The existence and uniqueness of solution of the fractional-order mechanical model are important and useful. Especially, by examining the numerical simulation and analysis of solution, one can undertake macroscopical analysis and comparative research into advantages and disadvantages of real estate securitization process and find that there may exist problems and risks with real estate asset securitization, and then one can put forward optimizing the views on traditional risk control process. In recent years, fractional-order models have been proved to be more accurate than integer order models, i.e., there are more degrees of freedom in the fractional-order models. Tao and Qian [24] discussed the existence and uniqueness of positive solutions for the following differential equation with nonlocal Riemann-Stieltjes integral condition arising from the real estate asset securitization:

$$
\left\{\begin{array}{l}
-D^{\alpha} x(t)=f\left(t, x(t),-D^{\beta} x(t)\right), \quad 0<t<1,2<\alpha \leq 3 \\
D^{\beta} x(0)=D^{\beta+1} x(0)=0, \quad D^{\beta} x(1)=\int_{0}^{1} D^{\beta} x(s) d A(s) .
\end{array}\right.
$$

Mathematical models have been proven valuable in understanding the dynamics of HIV infection [25-27]. Recently Perelson [28] introduced fractional order into a model of HIV infection. Motivated by these HIV models, Yang [29] considered the existence of nontrivial solution for the fractional differential system

$$
\left\{\begin{array}{l}
-D^{\alpha} x(t)=\lambda f\left(t, x(t),-D^{\beta} x(t), y(t)\right), \quad-D^{\gamma} y(t)=g(t, x(t)), \quad t \in(0,1), \\
D^{\beta} x(0)=0, \quad D^{\beta} x(1)=\int_{0}^{1} D^{\beta} x(s) d A(s), \\
y(0)=0, \quad y(1)=\int_{0}^{1} y(s) d B(s),
\end{array}\right.
$$

where $\lambda$ is a parameter, $1<\gamma<\alpha \leq 2,1<\alpha-\beta<\gamma, 0<\beta<1$ and $A, B$ are functions of bounded variation.

In the present paper, we are motivated by some recent papers $[18,24,29]$, which considered problem (1.1) with $F$ being single-valued and provided results on the existence and nonexistence of positive solutions. Here we extend the results to cover the multivalued case.

We establish existence results for problem (1.1), when the right-hand side is convex as well as non-convex valued. The first result relies on the nonlinear alternative of Leray-Schauder type. In the second result, we shall combine the nonlinear alternative of Leray-Schauder type for single-valued maps with a selection theorem due to Bressan and Colombo for lower semicontinuous multivalued maps with nonempty closed and decomposable values; while in the third result, we shall use the fixed point theorem for contraction multivalued maps due to Covitz and Nadler.

The paper is organized as follows. In Section 2 we recall some preliminary facts that we need in the sequel, and in Section 3 we prove our main results. Examples illustrating the obtained results are presented in Section 4. 


\section{Preliminaries}

In this section, we introduce notations, definitions and preliminary facts that will be used in the remainder of this paper.

Let $C([0,1])$ denote a Banach space of continuous functions from $[0,1]$ into $\mathbb{R}$ with the norm $\|x\|=\sup _{t \in[0,1]}|x(t)|$. Let $L^{1}([0,1], \mathbb{R})$ be the Banach space of measurable functions $x:[0,1] \rightarrow \mathbb{R}$ which are Lebesgue integrable and normed by $\|x\|_{L^{1}}=\int_{0}^{1}|x(t)| d t$.

For a normed space $(X,\|\cdot\|)$, let

$$
\begin{aligned}
& \mathcal{P}_{c l}(X)=\{Y \in \mathcal{P}(X): Y \text { is closed }\}, \\
& \mathcal{P}_{b}(X)=\{Y \in \mathcal{P}(X): Y \text { is bounded }\}, \\
& \mathcal{P}_{c p}(X)=\{Y \in \mathcal{P}(X): Y \text { is compact }\} \text { and } \\
& \mathcal{P}_{c p, c}(X)=\{Y \in \mathcal{P}(X): Y \text { is compact and convex }\} .
\end{aligned}
$$

Let $A, B \in \mathcal{P}_{c l}(X)$. The Pompeiu-Hausdorff distance of $A, B$ is defined by

$$
H_{d}(A, B)=\max \left\{\sup _{a \in A} d(a, B), \sup _{b \in B} d(A, b)\right\},
$$

where $d(A, b)=\inf _{a \in A} d(a ; b)$ and $d(a, B)=\inf _{b \in B} d(a ; b)$.

A multivalued map $G: X \rightarrow \mathcal{P}(X)$ :

(i) is convex (closed) valued if $G(x)$ is convex (closed) for all $x \in X$;

(ii) is bounded on bounded sets if $G(\mathbb{B})=\bigcup_{x \in \mathbb{B}} G(x)$ is bounded in $X$ for all $\mathbb{B} \in \mathcal{P}_{b}(X)$ (i.e., $\left.\sup _{x \in \mathbb{B}}\{\sup \{|y|: y \in G(x)\}\}<\infty\right)$;

(iii) is called upper semicontinuous (u.s.c.) on $X$ if, for each $x_{0} \in X$, the set $G\left(x_{0}\right)$ is a nonempty closed subset of $X$, and if, for each open set $N$ of $X$ containing $G\left(x_{0}\right)$, there exists an open neighborhood $\mathcal{N}_{0}$ of $x_{0}$ such that $G\left(\mathcal{N}_{0}\right) \subseteq N$;

(iv) $G$ is lower semicontinuous (l.s.c.) if the set $\{y \in X: G(y) \cap B \neq \emptyset\}$ is open for any open set $B$ in $E$;

(v) is said to be completely continuous if $G(\mathbb{B})$ is relatively compact for every $\mathbb{B} \in \mathcal{P}_{b}(X)$;

(vi) is said to be measurable if, for every $y \in \mathbb{R}$, the function

$$
t \mapsto d(y, G(t))=\inf \{|y-z|: z \in G(t)\}
$$

is measurable;

(vii) has a fixed point if there is $x \in X$ such that $x \in G(x)$. The fixed point set of the multivalued operator $G$ will be denoted by Fix $G$.

Definition 2.1 A multivalued operator $N: X \rightarrow \mathcal{P}_{c l}(X)$ is called:

(a) $\gamma$-Lipschitz if and only if there exists $\gamma>0$ such that

$$
H_{d}(N(x), N(y)) \leq \gamma d(x, y) \quad \text { for each } x, y \in X
$$

(b) a contraction if and only if it is $\gamma$-Lipschitz with $\gamma<1$.

Definition 2.2 A multivalued map $F:[0,1] \times \mathbb{R} \rightarrow \mathcal{P}(\mathbb{R})$ is said to be Carathéodory if (i) $t \mapsto F(t, x)$ is measurable for each $x \in \mathbb{R}$; 
(ii) $x \mapsto F(t, x)$ is upper semicontinuous for almost all $t \in[0,1]$. Further, a Carathéodory function $F$ is called $L^{1}$-Carathéodory if

(iii) for each $\alpha>0$, there exists $\varphi_{\alpha} \in L^{1}\left([0,1], \mathbb{R}^{+}\right)$such that

$$
\|F(t, x)\|=\sup \{|v|: v \in F(t, x)\} \leq \varphi_{\alpha}(t)
$$

for all $\|x\| \leq \alpha$ and for a.e. $t \in[0,1]$.

For each $x \in C([0,1], \mathbb{R})$, define the set of selections of $F$ by

$$
S_{F, x}:=\left\{v \in L^{1}([0,1], \mathbb{R}): v(t) \in F\left(t, x(t),-D^{\beta} x(t)\right) \text { for a.e. } t \in[0,1]\right\} .
$$

We define the graph of $G$ to be the set $\operatorname{Gr}(G)=\{(x, y) \in X \times Y, y \in G(x)\}$ and recall two useful results regarding closed graphs and upper-semicontinuity.

Lemma 2.3 [30, Proposition 1.2] If $G: X \rightarrow \mathcal{P}_{c l}(Y)$ is u.s.c., then $\operatorname{Gr}(G)$ is a closed subset of $X \times Y$; i.e., for every sequence $\left\{x_{n}\right\}_{n \in \mathbb{N}} \subset X$ and $\left\{y_{n}\right\}_{n \in \mathbb{N}} \subset Y$, if when $n \rightarrow \infty, x_{n} \rightarrow x_{*}$, $y_{n} \rightarrow y_{*}$ and $y_{n} \in G\left(x_{n}\right)$, then $y_{*} \in G\left(x_{*}\right)$. Conversely, if $G$ is completely continuous and has a closed graph, then it is upper semicontinuous.

Lemma 2.4 [31] Let $X$ be a Banach space. Let $F:[0, T] \times \mathbb{R} \rightarrow \mathcal{P}_{c p, c}(X)$ be an $L^{1}$ Carathéodory multivalued map and let $\Theta$ be a linear continuous mapping from $L^{1}([0,1], X)$ to $C([0,1], X)$. Then the operator

$$
\Theta \circ S_{F}: C([0,1], X) \rightarrow \mathcal{P}_{c p, c}(C([0,1], X)), \quad x \mapsto\left(\Theta \circ S_{F}\right)(x)=\Theta\left(S_{F, x, y}\right)
$$

is a closed graph operator in $C([0,1], X) \times C([0,1], X)$.

Let us recall some basic definitions of fractional calculus $[1,2]$.

Definition 2.5 The Riemann-Liouville derivative of fractional order $q$ is defined as

$$
D_{0+}^{q} g(t)=\frac{1}{\Gamma(n-q)}\left(\frac{d}{d t}\right)^{n} \int_{0}^{t}(t-s)^{n-q-1} g(s) d s, \quad n-1<q<n, n=[q]+1,
$$

provided the integral exists, where $[q]$ denotes the integer part of the real number $q$.

Definition 2.6 The Riemann-Liouville fractional integral of order $q$ is defined as

$$
I_{0+}^{q} g(t)=\frac{1}{\Gamma(q)} \int_{0}^{t} \frac{g(s)}{(t-s)^{1-q}} d s, \quad q>0,
$$

provided the integral exists.

Lemma 2.7 [2] For $q>0$, the general solution of the fractional differential equation $D_{0+}^{q} x(t)=0$ is given by

$$
x(t)=c_{1} t^{q-1}+c_{2} t^{q-2}+\cdots+c_{n} t^{q-n},
$$

where $c_{i} \in \mathbb{R}, i=1,2, \ldots, n(n=[q]+1)$. 
Lemma 2.8 [2] (1) If $x \in L^{1}(0,1), v>\sigma>0$, then

$$
I^{\nu} I^{\sigma} x(t)=I^{\nu+\sigma} x(t), \quad D^{\sigma} I^{\sigma} x(t)=I^{\nu-\sigma} x(t), \quad D^{\sigma} I^{\sigma} x(t)=x(t) .
$$

(2) If $v>0, \sigma>0$, then

$$
D^{v} t^{\sigma-1}=\frac{\Gamma(\sigma)}{\Gamma(\sigma-v)} t^{\sigma-v-1} .
$$

In view of Lemma 2.7, it follows that

$$
I_{0+}^{q} D_{0+}^{q} x(t)=x(t)+c_{1} t^{q-1}+c_{2} t^{q-2}+\cdots+c_{n} t^{q-n}
$$

for some $c_{i} \in \mathbb{R}, i=1,2, \ldots, n(n=[q]+1)$.

Let $x(t)=I^{\beta} y(t), y(t) \in C[0,1]$. By standard discussion, one can easily reduce BVP (1.1) to the following modified problem:

$$
\left\{\begin{array}{l}
-D^{\alpha-\beta} y(t) \in F\left(t, I^{\beta} y(t),-y(t)\right), \quad 0<t<1,2<\alpha \leq 3 \\
y(0)=y^{\prime}(0)=0, \quad y(1)=\int_{0}^{1} y(s) d A(s)
\end{array}\right.
$$

and BVP (2.4) is equivalent to BVP (1.1).

Lemma 2.9 $[19]$ Given $y \in L^{1}([0,1], \mathbb{R})$, then the unique solution of the problem

$$
\left\{\begin{array}{l}
D^{\alpha-\beta} x(t)+y(t)=0, \quad 0<t<1, \\
x(0)=x^{\prime}(0)=0, \quad x(1)=0,
\end{array}\right.
$$

is given by

$$
x(t)=\int_{0}^{1} G(t, s) y(s) d s
$$

where $G(t, s)$ is the Green function of $B V P(2.5)$ and is given by

$$
G(t, s)= \begin{cases}\frac{[t(1-s)]^{\alpha-\beta-1}}{\Gamma(\alpha-\beta)}, & 0 \leq t \leq s \leq 1 \\ \frac{[t(1-s)]^{\alpha-\beta-1}-(t-s)^{\alpha-\beta-1}}{\Gamma(\alpha-\beta)}, & 0 \leq s \leq t \leq 1 .\end{cases}
$$

Lemma 2.10 For any $t, s \in[0,1], G(t, s)$ satisfies

$$
\frac{t^{\alpha-\beta-1}(1-t) s(1-s)^{\alpha-\beta-1}}{\Gamma(\alpha-\beta)} \leq G(t, s) \leq \frac{s(1-s)^{\alpha-\beta-1}}{\Gamma(\alpha-\beta-1)} .
$$

By Lemma 2.9, the unique solution of the problem

$$
\left\{\begin{array}{l}
D^{\alpha} x(t)=0, \quad 0<t<1, \\
x(0)=x^{\prime}(0)=0, \quad x(1)=1,
\end{array}\right.
$$


is $t^{\alpha-\beta-1}$. Let

$$
\mathcal{C}=\int_{0}^{1} t^{\alpha-\beta-1} d A(t)
$$

and define

$$
\mathcal{G}_{A}(s)=\int_{0}^{1} G(t, s) d A(t)
$$

as in [19], we can get that the Green function for BVP (2.4) is given by

$$
H(t, s)=\frac{t^{\alpha-\beta-1}}{1-\mathcal{C}} \mathcal{G}_{A}(s)+G(t, s) .
$$

Throughout the paper we always assume that the following holds.

$\left(\mathrm{H}_{0}\right) A$ is an increasing function of bounded variation such that $\mathcal{G}(s) \geq 0$ for $s \in[0,1]$ and $0 \leq \mathcal{C}<1$, where $\mathcal{C}$ is defined by (2.9).

Lemma 2.11 [21] Let $1<\alpha-\beta \leq 2$. Assume that $\left(\mathrm{H}_{0}\right)$ holds. Then $H(t, s)$ satisfies

$$
0 \leq H(t, s) \leq \frac{1}{(1-\mathcal{C}) \Gamma(\alpha-\beta-1)}
$$

We end this section by recalling two well-known fixed point theorems which will be used in the sequel, the nonlinear alternative of Leray-Schauder for multivalued maps and Covitz and Nadler fixed point theorem.

Lemma 2.12 (Nonlinear alternative for Kakutani maps) [32] Let E be a Banach space, let $C$ be a closed convex subset of $E, U$ be an open subset of $C$ and $0 \in U$. Suppose that $F: \bar{U} \rightarrow \mathcal{P}_{c, c v}(C)$ is an upper semicontinuous compact map. Then either

(i) F has a fixed point in $\bar{U}$, or

(ii) there is $a u \in \partial U$ and $\lambda \in(0,1)$ with $u \in \lambda F(u)$.

Lemma 2.13 [33] Let $(X, d)$ be a complete metric space. If $N: X \rightarrow \mathcal{P}_{c l}(X)$ is a contraction, then Fix $N \neq \emptyset$.

\section{Existence results}

Now we are in a position to present our main results. The methods used to prove the existence results are standard; however, their exposition in the framework of problem (1.1) is new.

\subsection{Convex case}

Theorem 3.1 Assume that $\left(\mathrm{H}_{0}\right)$ holds. In addition we assume that:

$\left(\mathrm{H}_{1}\right) \quad F:[0,1] \times \mathbb{R} \times \mathbb{R} \rightarrow \mathcal{P}(\mathbb{R})$ is Carathéodory and has nonempty compact and convex values; 
$\left(\mathrm{H}_{2}\right)$ there exist two continuous nondecreasing functions $\psi_{i}:[0, \infty) \rightarrow(0, \infty), i=1,2$, and a function $p \in L^{1}\left([0,1], \mathbb{R}^{+}\right)$such that

$$
\|F(t, x, y)\|_{\mathcal{P}}:=\sup \{|z|: z \in F(t, x, y)\} \leq p(t)\left(\psi_{1}(|x|)+\psi_{2}(|y|)\right)
$$

for each $(t, x, y) \in[0,1] \times \mathbb{R} \times \mathbb{R}$;

$\left(\mathrm{H}_{3}\right)$ there exists a constant $M>0$ such that

$$
\frac{M}{\frac{1}{(1-\mathcal{C}) \Gamma(\alpha-\beta-1)}\left[\psi_{1}\left(\frac{M}{\Gamma(\beta+1)}\right)+\psi_{2}(M)\right]\|p\|_{L^{1}}}>1 .
$$

Then boundary value problem (1.1) has at least one solution on $[0,1]$.

Proof Define the operator $\Omega_{F}: C([0,1], \mathbb{R}) \rightarrow \mathcal{P}(C([0,1], \mathbb{R}))$ by

$$
\Omega_{F}(x)=\left\{h \in C([0,1], \mathbb{R}): h(t)=\int_{0}^{1} H(t, s) v(s) d s, v \in S_{F, x}\right\} .
$$

We will show that $\Omega_{F}$ satisfies the assumptions of the nonlinear alternative of LeraySchauder type. The proof consists of several steps. As the first step, we show that $\Omega_{F}$ is convex for each $x \in C([0,1], \mathbb{R})$. This step is obvious since $S_{F, x, y}$ is convex ( $F$ has convex values), and therefore we omit the proof.

In the second step, we show that $\Omega_{F}$ maps bounded sets (balls) into bounded sets in $C([0,1], \mathbb{R})$. For a positive number $\rho$, let $B_{\rho}=\{x \in C([0,1], \mathbb{R}):\|x\| \leq \rho\}$ be a bounded ball in $C([0,1], \mathbb{R})$. Then, for each $h \in \Omega_{F}(x), x \in B_{\rho}$, there exists $v \in S_{F, x, y}$ such that

$$
h(t)=\int_{0}^{1} H(t, s) v(s) d s
$$

Then for $t \in[0,1]$, and notice that

$$
\left|I^{\beta} y(t)\right|=\left|\frac{1}{\Gamma(\beta)} \int_{0}^{t}(t-s)^{\beta-1} y(s) d s\right| \leq \frac{\|y\|}{\Gamma(\beta+1)},
$$

we have

$$
\begin{aligned}
|h(t)| & \leq \int_{0}^{1} H(t, s)|v(s)| d s \\
& \leq \frac{1}{(1-\mathcal{C}) \Gamma(\alpha-\beta-1)} \int_{0}^{1} p(s)\left[\psi_{1}\left(\left|I^{\beta} y(s)\right|\right)+\psi_{2}(|y(s)|)\right] d s \\
& \leq \frac{1}{(1-\mathcal{C}) \Gamma(\alpha-\beta-1)} \int_{0}^{1} p(s)\left[\psi_{1}\left(\frac{\|y\|}{\Gamma(\beta+1)}\right)+\psi_{2}(\|y\|)\right] \\
& \leq \frac{1}{(1-\mathcal{C}) \Gamma(\alpha-\beta-1)}\left[\psi_{1}\left(\frac{\|y\|}{\Gamma(\beta+1)}\right)+\psi_{2}(\|y\|)\right] \int_{0}^{1} p(s) d s .
\end{aligned}
$$

Thus,

$$
\|h\| \leq \frac{1}{(1-\mathcal{C}) \Gamma(\alpha-\beta-1)}\left[\psi_{1}\left(\frac{\rho}{\Gamma(\beta+1)}\right)+\psi_{2}(\rho)\right]\|p\|_{L^{1}} .
$$


Now we show that $\Omega_{F}$ maps bounded sets into equicontinuous sets of $C([0,1], \mathbb{R})$. Let $t^{\prime}, t^{\prime \prime} \in[0,1]$ with $t^{\prime}<t^{\prime \prime}$ and $y \in B_{\rho}$. For each $h \in \Omega_{F}(y)$, we obtain

$$
\begin{aligned}
\left|h\left(t^{\prime \prime}\right)-h\left(t^{\prime}\right)\right| & \leq \int_{0}^{1}\left|H\left(t^{\prime}, s\right)-H\left(t^{\prime \prime}, s\right)\right||v(s)| d s \\
& \leq \int_{0}^{1}\left|H\left(t^{\prime}, s\right)-H\left(t^{\prime \prime}, s\right)\right| p(s)\left[\psi\left(\frac{\rho}{\Gamma(\beta+1)}\right)+\psi(\rho)\right] d s .
\end{aligned}
$$

Obviously the right-hand side of the above inequality tends to zero independently of $y \in B_{\rho}$ as $t^{\prime \prime}-t^{\prime} \rightarrow 0$. As $\Omega_{F}$ satisfies the above three assumptions, it follows by the ArzeláAscoli theorem that $\Omega_{F}: C([0,1], \mathbb{R}) \rightarrow \mathcal{P}(C([0,1], \mathbb{R}))$ is completely continuous.

In our next step, we show that $\Omega_{F}$ has a closed graph. Let $y_{n} \rightarrow y_{*}, h_{n} \in \Omega_{F}\left(y_{n}\right)$ and $h_{n} \rightarrow h_{*}$. Then we need to show that $h_{*} \in \Omega_{F}\left(y_{*}\right)$. Associated with $h_{n} \in \Omega_{F}\left(y_{n}\right)$, there exists $v_{n} \in S_{F, y_{n}}$ such that for each $t \in[0,1]$,

$$
h_{n}(t)=\int_{0}^{1} H(t, s) v_{n}(s) d s
$$

Thus it suffices to show that there exists $v_{*} \in S_{F, y_{*}}$ such that for each $t \in[0,1]$,

$$
h_{*}(t)=\int_{0}^{1} H(t, s) v_{*}(s) d s
$$

Let us consider the linear operator $\Theta: L^{1}([0,1], \mathbb{R}) \rightarrow C([0,1], \mathbb{R})$ given by

$$
f \mapsto \Theta(v)(t)=\int_{0}^{1} H(t, s) v(s) d s .
$$

Observe that

$$
\left\|h_{n}(t)-h_{*}(t)\right\|=\left\|\int_{0}^{1} H(t, s)\left(v_{n}(s)-v_{*}(s)\right) d s\right\| \rightarrow 0, \quad \text { as } n \rightarrow \infty .
$$

Thus, it follows by Lemma 2.4 that $\Theta \circ S_{F}$ is a closed graph operator. Further, we have $h_{n}(t) \in \Theta\left(S_{F, y_{n}}\right)$. Since $y_{n} \rightarrow y_{*}$, therefore, we have

$$
h_{*}(t)=\int_{0}^{1} H(t, s) v_{*}(s) d s
$$

for some $v_{*} \in S_{F, y_{*}}$.

Finally, we show that there exists an open set $U \subseteq C([0,1], \mathbb{R})$ with $y \notin \Omega_{F}(y)$ for any $\lambda \in(0,1)$ and all $y \in \partial U$. Let $\lambda \in(0,1)$ and $y \in \lambda \Omega_{F}(y)$. Then there exists $v \in L^{1}([0,1], \mathbb{R})$ with $v \in S_{F, y}$ such that, for $t \in[0,1]$, we have

$$
y(t)=\lambda \int_{0}^{1} H(t, s) v(s) d s
$$


and, using the computations of the second step above, we have

$$
\begin{aligned}
|y(t)| & \leq \frac{1}{(1-\mathcal{C}) \Gamma(\alpha-\beta-1)} \int_{0}^{1} p(s)\left[\psi_{1}\left(\left|I^{\beta} y(s)\right|\right)+\psi_{2}(|y(s)|)\right] d s \\
& \leq \frac{1}{(1-\mathcal{C}) \Gamma(\alpha-\beta-1)}\left[\psi_{1}\left(\frac{\|y\|}{\Gamma(\beta+1)}\right)+\psi_{2}(\|y\|)\right]\left\|p_{1}\right\|_{L^{1}} .
\end{aligned}
$$

Consequently, we have

$$
\frac{\|y\|}{\frac{1}{(1-\mathcal{C}) \Gamma(\alpha-\beta-1)}\left[\psi_{1}\left(\frac{\|y\|}{\Gamma(\beta+1)}\right)+\psi_{2}(\|y\|)\right]\|p\|_{L^{1}}} \leq 1 .
$$

In view of $\left(\mathrm{H}_{3}\right)$, there exists $M$ such that $\|y\| \neq M$. Let us set

$$
U=\{y \in C([0,1], \mathbb{R}):\|y\|<M\} .
$$

Note that the operator $\Omega_{F}: \bar{U} \rightarrow \mathcal{P}(C([0,1], \mathbb{R}))$ is upper semicontinuous and completely continuous. From the choice of $U$, there is no $y \in \partial U$ such that $y \in \lambda \Omega_{F}(y)$ for some $\lambda \in$ $(0,1)$. Consequently, by the nonlinear alternative of Leray-Schauder type (Lemma 2.12), we deduce that $\Omega_{F}$ has a fixed point $y \in \bar{U}$, which is a solution of problem (1.1). This completes the proof.

\subsection{Non-convex case}

In this subsection, we study the case when $F$ is not necessarily convex-valued.

Definition 3.2 Let $A$ be a subset of $[0,1] \times \mathbb{R}$. $A$ is $\mathcal{L} \otimes \mathcal{B}$ measurable if $A$ belongs to the $\sigma$-algebra generated by all sets of the form $\mathcal{J} \times \mathcal{D}$, where $\mathcal{J}$ is Lebesgue measurable in $[0,1]$ and $\mathcal{D}$ is Borel measurable in $\mathbb{R}$.

Definition 3.3 A subset $\mathcal{A}$ of $L^{1}([0,1], \mathbb{R})$ is decomposable if, for all $u, v \in \mathcal{A}$ and measurable $\mathcal{J} \subset[0,1]=J$, the function $u \chi_{\mathcal{J}}+v \chi_{J-\mathcal{J}} \in \mathcal{A}$, where $\chi_{\mathcal{J}}$ stands for the characteristic function of $\mathcal{J}$.

Theorem 3.4 Assume that $\left(\mathrm{H}_{0}\right),\left(\mathrm{H}_{2}\right),\left(\mathrm{H}_{3}\right)$ and the following condition holds:

$\left(\mathrm{H}_{4}\right) \quad F:[0,1] \times \mathbb{R} \times \mathbb{R} \rightarrow \mathcal{P}(\mathbb{R})$ is a nonempty compact-valued multivalued map such that

(a) $(t, x, y) \mapsto F(t, x, y)$ is $\mathcal{L} \otimes \mathcal{B} \otimes \mathcal{B}$ measurable,

(b) $(x, y) \mapsto F(t, x, y)$ is lower semicontinuous for each $t \in[0,1]$.

Then boundary value problem (1.1) has at least one solution on $[0,1]$.

Proof It follows from $\left(\mathrm{H}_{2}\right),\left(\mathrm{H}_{4}\right)$ and Lemma 4.1 of [34] that $F$ is of l.s.c. type. Then, from the selection theorem due to Bressan and Colombo [35] for lower semicontinuous maps with decomposable values, there exists a continuous function $f: A C^{2}([0,1], \mathbb{R}) \rightarrow$ $L^{1}([0,1], \mathbb{R})$ such that $f(x) \in \mathcal{F}(x)$ for all $x \in C([0,1], \mathbb{R})$.

Consider the problem

$$
\left\{\begin{array}{l}
-D^{\alpha} x(t)=f(x(t)), \quad t \in[0,1], \\
D^{\beta} x(0)=D^{\beta+1} x(0)=0, \quad D^{\beta} x(1)=\int_{0}^{1} D^{\beta} x(s) d A(s) .
\end{array}\right.
$$


Observe that if $x \in A C^{2}([0,1], \mathbb{R})$ is a solution of (3.1), then $x$ is a solution to problem (1.1). In order to transform problem (3.1) into a fixed point problem, we define the operator $\bar{\Omega}_{F}$ as

$$
\bar{\Omega}_{F} x(t)=\int_{0}^{1} H(t, s) f(x(s)) d s
$$

It can easily be shown that $\bar{\Omega}_{F}$ is continuous and completely continuous and satisfies all the conditions of the nonlinear alternative of Leray-Schauder type for single-valued maps [32]. The remaining part of the proof is similar to that of Theorem 3.1. So we omit it. This completes the proof.

Now we prove our second existence result for problem (1.1) with a non-convex-valued right-hand side by applying a fixed point theorem for a multivalued map due to Covitz and Nadler [33].

Theorem 3.5 Assume that the following conditions hold:

$\left(\mathrm{H}_{5}\right) \quad F:[0,1] \times \mathbb{R} \times \mathbb{R} \rightarrow \mathcal{P}_{c p}(\mathbb{R})$ is such that $F(\cdot, x, y):[0,1] \rightarrow \mathcal{P}_{c p}(\mathbb{R})$ is measurable for each $x \in \mathbb{R}$

$\left(\mathrm{H}_{6}\right) H_{d}(F(t, x, y), F(t, \bar{x}, \bar{y})) \leq m(t)(|x-\bar{x}|+|y-\bar{y}|)$ for almost all $t \in[0,1]$ and $x, \bar{x}, y, \bar{y} \in \mathbb{R}$ with $m \in L^{1}\left([0,1], \mathbb{R}^{+}\right)$and $d(0, F(t, 0,0)) \leq m(t)$ for almost all $t \in[0,1]$.

Then boundary value problem (1.1) has at least one solution on $[0,1]$ if

$$
\frac{1}{(1-\mathcal{C}) \Gamma(\alpha-\beta-1)}\left(\frac{1}{\Gamma(\beta+1)}+1\right)\|m\|_{L^{1}}<1 .
$$

Proof We transform boundary value problem (1.1) into a fixed point problem. Define the operator $\Omega_{F}: C([0,1], \mathbb{R}) \rightarrow \mathcal{P}(C([0,1], \mathbb{R}))$ by

$$
\Omega_{F}(x)=\left\{h \in C([0,1], \mathbb{R}): h(t)=\int_{0}^{1} H(t, s) v(s) d s, v \in S_{F, x}\right\} .
$$

We show that the operator $\Omega_{F}$ satisfies the assumptions of Lemma 2.13. The proof will be given in two steps.

Step 1. $\Omega_{F}(x)$ is nonempty and closed for every $v \in S_{F, x}$. Note that since the set-valued map $F(\cdot, x(\cdot), y(\cdot))$ is measurable with the measurable selection theorem $(e . g$., [36, Theorem III.6]), it admits a measurable selection $v: I \rightarrow \mathbb{R}$. Moreover, by the assumption $\left(\mathrm{H}_{5}\right)$, we have

$$
|v(t)| \leq m(t)+m(t)\left(|y(t)|+\left|I^{\beta} y(t)\right|\right) \leq m(t)+m(t)\left(\frac{1}{\Gamma(\beta+1)}+1\right)|y(t)|,
$$

i.e., $v \in L^{1}([0,1], \mathbb{R})$ and hence $F$ is integrably bounded. Therefore, $S_{F, y} \neq \emptyset$.

To show that $\Omega_{F}(x) \in \mathcal{P}_{c l}((C[0,1], \mathbb{R}))$ for each $x \in C([0,1], \mathbb{R})$, let $\left\{u_{n}\right\}_{n \geq 0} \in \Omega_{F}(x)$ be such that $u_{n} \rightarrow u(n \rightarrow \infty)$ in $C([0,1], \mathbb{R})$. Then $u \in C([0,1], \mathbb{R})$ and there exists $v_{n} \in S_{F, x_{n}}$ such that, for each $t \in[0,1]$,

$$
u_{n}(t)=\int_{0}^{1} H(t, s) v_{n}(s) d s
$$


As $F$ has compact values, we pass onto a subsequence (if necessary) to obtain that $v_{n}$ converges to $v$ in $L^{1}([0,1], \mathbb{R})$. Thus, $v \in S_{F, y}$ and for each $t \in[0,1]$, we have

$$
v_{n}(t) \rightarrow v(t)=\int_{0}^{1} H(t, s) v(s) d s .
$$

Hence, $u \in \Omega_{F}(x)$.

Step 2. The multivalued map $\Omega_{F}(x)$ is a contraction. We show that there exists $\delta<1$ such that

$$
H_{d}\left(\Omega_{F}(x), \Omega_{F}(\bar{x})\right) \leq \delta\|x-\bar{x}\| \quad \text { for each } x, \bar{x} \in A C^{1}([0,1], \mathbb{R}) .
$$

Let $x, \bar{x} \in A C^{1}([0,1], \mathbb{R})$ and $h_{1} \in \Omega_{F}(x)$. Then there exists $v_{1}(t) \in F(t, y(t))$ such that, for each $t \in[0,1]$,

$$
h_{1}(t)=\int_{0}^{1} H(t, s) v_{1}(s) d s
$$

By $\left(\mathrm{H}_{6}\right)$, we have

$$
H_{d}(F(t, x, y), F(t, \bar{x}, \bar{y})) \leq m(t)(|x(t)-\bar{x}(t)|+|y(t)-\bar{y}(t)|) .
$$

So, there exists $w(t) \in F(t, \bar{x}(t), \bar{y}(t))$ such that

$$
\left|v_{1}(t)-w(t)\right| \leq m(t)(|x(t)-\bar{x}(t)|+|y(t)-\bar{y}(t)|), \quad t \in[0,1] .
$$

Define $U:[0,1] \rightarrow \mathcal{P}(\mathbb{R})$ by

$$
U(t)=\left\{w \in \mathbb{R}:\left|v_{1}(t)-w(t)\right| \leq m(t)(|x(t)-\bar{x}(t)|+|y(t)-\bar{y}(t)|)\right\} .
$$

Since the multivalued operator $U(t) \cap F(t, \bar{x}(t), \bar{y}(t))$ is measurable (Proposition III.4 in [36]), there exists a function $v_{2}(t)$ which is a measurable selection for $U$. So, $v_{2}(t) \in$ $F(t, \bar{x}(t), \bar{y}(t))$ and for each $t \in[0,1]$, we have $\left|v_{1}(t)-v_{2}(t)\right| \leq m(t)(|x(t)-\bar{x}(t)|+|y(t)-\bar{y}(t)|)$.

For each $t \in[0,1]$, let us define

$$
h_{2}(t)=\int_{0}^{1} H(t, s) v_{2}(s) d s
$$

Thus,

$$
\begin{aligned}
\left|h_{1}(t)-h_{2}(t)\right| & \leq \int_{0}^{1} H(t, s)\left|v_{1}(s)-v_{2}(s)\right| d s \\
& \leq \frac{1}{(1-\mathcal{C}) \Gamma(\alpha-\beta-1)}\left(\frac{1}{\Gamma(\beta+1)}+1\right)\|x-\bar{x}\| \int_{0}^{1} m(s) d s .
\end{aligned}
$$

Hence,

$$
\left\|h_{1}-h_{2}\right\| \leq \frac{1}{(1-\mathcal{C}) \Gamma(\alpha-\beta-1)}\left(\frac{1}{\Gamma(\beta+1)}+1\right)\|m\|_{L^{1}}\|x-\bar{x}\| .
$$


Analogously, interchanging the roles of $x$ and $\bar{x}$, we obtain

$$
H_{d}\left(\Omega_{F}(x), \Omega_{F}(\bar{x})\right) \leq \delta\|x-\bar{x}\| \leq \frac{1}{(1-\mathcal{C}) \Gamma(\alpha-\beta-1)}\left(\frac{1}{\Gamma(\beta+1)}+1\right)\|m\|_{L^{1}}\|x-\bar{x}\| .
$$

Since $\Omega_{F}$ is a contraction, it follows by Lemma 2.13 that $\Omega_{F}$ has a fixed point $x$ which is a solution of (1.1). This completes the proof.

\section{Examples}

In this section, we give two examples to show the applicability of our results.

Example 4.1 Consider the following fractional boundary value problem:

$$
\left\{\begin{array}{l}
-D^{5 / 2} x(t) \in F\left(t, x(t),-D^{1 / 8} x(t)\right), \quad t \in[0,1] \\
D^{1 / 8} x(0)=D^{9 / 8} x(0)=0, \quad D^{1 / 8} x(1)=\int_{0}^{1} D^{1 / 8} x(s) d A(s),
\end{array}\right.
$$

where

$$
A(t)= \begin{cases}0, & t \in\left[0, \frac{1}{4}\right), \\ 1, & t \in\left[\frac{1}{4}, \frac{3}{4}\right), \\ 2, & t \in\left[\frac{3}{4}, 1\right]\end{cases}
$$

and $F:[0,1] \times \mathbb{R} \times \mathbb{R} \rightarrow \mathcal{P}(\mathbb{R})$ is a multivalued map given by

$$
F(t, x, y)=\left\{u \in \mathbb{R}: e^{-|x|}-\frac{|y|}{1+|y|}+\sin t \leq u \leq 5+\frac{|x|}{1+x^{2}}+6 t^{3}+\cos y\right\} .
$$

Thus BVP (4.1) becomes the four-point BVP

$$
\left\{\begin{array}{l}
-D^{5 / 2} x(t) \in F\left(t, x(t),-D^{1 / 8} x(t)\right), \quad t \in[0,1] \\
D^{1 / 8} x(0)=D^{9 / 8} x(0)=0, \quad D^{1 / 8} x(1)=D^{1 / 8} x\left(\frac{1}{4}\right)+D^{1 / 8} x\left(\frac{3}{4}\right) .
\end{array}\right.
$$

We have

$$
\begin{aligned}
& 0 \leq \mathcal{C}=\int_{0}^{1} t^{3 / 2} d A(t)=\left(\frac{1}{4}\right)^{3 / 2}+\left(\frac{3}{4}\right)^{3 / 2} \approx 0.7745, \\
& (1-\mathcal{C}) \Gamma(\alpha-\beta-1)=0.2014, \\
& G(t, s)= \begin{cases}G_{1}(t, s)=\frac{[t(1-s)]^{3 / 2}}{\Gamma(5 / 2)}, & 0 \leq t \leq s \leq 1, \\
G_{2}(t, s)=\frac{[t(1-s)]^{3 / 2}-(t-s)^{3 / 2}}{\Gamma(5 / 2)}, & 0 \leq s \leq t \leq 1\end{cases}
\end{aligned}
$$

and

$$
\mathcal{G}_{A}(s)= \begin{cases}G_{2}\left(\frac{1}{4}, s\right)+2 G_{2}\left(\frac{3}{4}, s\right), & 0 \leq s<\frac{1}{4}, \\ G_{1}\left(\frac{1}{4}, s\right)+2 G_{2}\left(\frac{3}{4}, s\right), & \frac{1}{4} \leq s<\frac{3}{4} \\ G_{1}\left(\frac{1}{4}, s\right)+2 G_{1}\left(\frac{3}{4}, s\right), & \frac{3}{4} \leq s \leq 1\end{cases}
$$

Thus $\mathcal{G}_{A}(s) \geq 0,0 \leq \mathcal{C}<1$ and $A(s)$ is increasing, so $\left(\mathrm{H}_{0}\right)$ holds. 
Moreover,

$$
\|F(t, x, y)\|_{\mathcal{P}}:=\sup \{|v|: v \in F(t, x, y)\} \leq 7+6 t^{3} \leq 13, \quad t \in[0,1], x, y \in \mathbb{R} .
$$

It is clear that $F$ is convex, compact-valued and is of Carathéodory type. Let $p(t)=1$ and $\psi_{1}(|x|)=3, \psi_{2}(|y|)=10$, we get for $t \in[0,1], x, y \in \mathbb{R}$

$$
\|F(t, x, y)\|_{\mathcal{P}}:=\sup \{|v|: v \in F(t, x, y)\} \leq p(t)\left(\psi_{1}(|x|)+\psi_{2}(|y|)\right)
$$

and hence $\left(\mathrm{H}_{2}\right)$ holds.

Using the above values in the condition $\left(\mathrm{H}_{3}\right)$

$$
\frac{M}{\frac{1}{(1-\mathcal{C}) \Gamma(\alpha-\beta-1)}\left[\psi_{1}\left(\frac{M}{\Gamma(\beta+1)}\right)+\psi_{2}(M)\right]\|p\|_{L^{1}}}>1
$$

we find that

$$
M>64.548161 .
$$

Clearly, all the conditions of Theorem 3.1 are satisfied. Hence the conclusion of Theorem 3.1 applies to problem (4.1).

Example 4.2 Consider the following fractional boundary value problem:

$$
\left\{\begin{array}{l}
-D^{5 / 2} x(t) \in F\left(t, x(t),-D^{1 / 8} x(t)\right), \quad t \in[0,1], \\
D^{1 / 8} x(0)=D^{9 / 8} x(0)=0, \quad D^{1 / 8} x(1)=\int_{0}^{1} D^{1 / 8} x(s) d A(s),
\end{array}\right.
$$

where $A(t)$ as in Example 4.1 and

$$
F(t, x, y)=\left[-\ell_{1}(t)-\frac{\sin x}{(4+t)^{2}}-2,-\frac{1}{10}\right] \cup\left[0, \frac{1}{32} \frac{|y|}{1+|y|}+\ell_{2}(t)\right]
$$

and $\ell_{1}, \ell_{2} \in L^{1}\left([0,1], \mathbb{R}^{+}\right)$.

Then we have

$$
\begin{aligned}
& \sup \{|u|: u \in F(t, x, y)\} \leq 3+\frac{1}{(4+t)^{2}}+\ell_{1}(t)+\ell_{2}(t), \\
& H_{d}(F(t, x, y)-F(t, \bar{x}, \bar{y})) \leq \frac{1}{(4+t)^{2}}|x-\bar{x}|+\frac{1}{32}|y-\bar{y}| .
\end{aligned}
$$

Let $m(t)=\frac{1}{(4+t)^{2}}+\frac{1}{32}$. Then

$$
H_{d}(F(t, x, y)-F(t, \bar{x}, \bar{y})) \leq m(t)(|x-\bar{x}|+|y-\bar{y}|)
$$

and

$$
\frac{1}{(1-\mathcal{C}) \Gamma(\alpha-\beta-1)}\left(\frac{1}{\Gamma(\beta+1)}+1\right)\|m\|_{L^{1}} \approx 0.8381486<1 .
$$

Hence all the assumptions of Theorem 3.5 are satisfied and by the conclusion of it, BVP (4.3) has at least one solution on $[0,1]$. 


\section{Competing interests}

The authors declare that they have no competing interests.

\section{Authors' contributions}

Each of the authors, BA and SKN, contributed to each part of this work equally and read and approved the final version of the manuscript.

\section{Author details}

${ }^{1}$ Department of Mathematics, Faculty of Science, King Abdulaziz University, P.O. Box 80203, Jeddah, 21589, Saudi Arabia.

${ }^{2}$ Department of Mathematics, University of Ioannina, loannina, 451 10, Greece.

\section{Authors' information}

Sotiris K Ntouyas is a member of Nonlinear Analysis and Applied Mathematics (NAAM) - Research Group at King Abdulaziz University, Jeddah, Saudi Arabia.

\section{Acknowledgements}

The authors thank the reviewers for their useful comments. This paper was partially supported by the Deanship of Scientific Research (DSR), King Abdulaziz University, Jeddah, Saudi Arabia.

\section{Received: 3 May 2013 Accepted: 5 July 2013 Published: 22 July 2013}

\section{References}

1. Podlubny, I: Fractional Differential Equations. Academic Press, San Diego (1999)

2. Kilbas, AA, Srivastava, HM, Trujillo, JJ: Theory and Applications of Fractional Differential Equations. North-Holland Mathematics Studies, vol. 204. Elsevier, Amsterdam (2006)

3. Baleanu, D, Diethelm, K, Scalas, E, Trujillo, JJ: Fractional Calculus Models and Numerical Methods. Series on Complexity, Nonlinearity and Chaos. World Scientific, Boston (2012)

4. Agarwal, RP, Andrade, B, Cuevas, C: Weighted pseudo-almost periodic solutions of a class of semilinear fractional differential equations. Nonlinear Anal., Real World Appl. 11, 3532-3554 (2010)

5. Ahmad, B, Nieto, JJ: Existence results for nonlinear boundary value problems of fractional integrodifferential equations with integral boundary conditions. Bound. Value Probl. 2009, Article ID 708576 (2009)

6. Ahmad, B, Ntouyas, SK: Fractional differential inclusions with fractional separated boundary conditions. Fract. Calc. Appl. Anal. 15, 362-382 (2012)

7. Ahmad, B, Ntouyas, SK, Alsaedi, A: A nonlocal three-point inclusion problem of Langevin equation with two different fractional orders. Adv. Differ. Equ. 2012, 54 (2012)

8. Ahmad, B, Ntouyas, SK: Existence results for higher order fractional differential inclusions with multi-strip fractional integral boundary conditions. Electron. J. Qual. Theory Differ. Equ. 2013, 20 (2013)

9. Ahmad, B, Ntouyas, SK, Alsaedi, A: On fractional differential inclusions with anti-periodic type integral boundary conditions. Bound. Value Probl. 2013, 82 (2013)

10. Ahmad, B, Ntouyas, SK, Alsaedi, A: A study of nonlinear fractional differential equations of arbitrary order with Riemann-Liouville type multi-strip boundary conditions. Math. Probl. Eng. 2013, Article ID 320415 (2013)

11. Bai, ZB: On positive solutions of a nonlocal fractional boundary value problem. Nonlinear Anal. 72, $916-924$ (2010)

12. Balachandran, K, Trujillo, JJ: The nonlocal Cauchy problem for nonlinear fractional integrodifferential equations in Banach spaces. Nonlinear Anal. 72, 4587-4593 (2010)

13. Baleanu, D, Mustafa, OG: On the global existence of solutions to a class of fractional differential equations. Comput. Math. Appl. 59, 1835-1841 (2010)

14. Baleanu, D, Mustafa, OG, O'Regan, D: A Nagumo-like uniqueness theorem for fractional differential equations. J. Phys. A, Math. Theor. 44(39), Article ID 392003 (2011)

15. El-Shahed, M, Shammakh, WM: Multiple positive solutions for nonlinear fractional eigenvalue problem with non local conditions. Fract. Calc. Appl. Anal. 3, 1-13 (2012)

16. Nyamoradi, N, Baleanu, D, Agarwal, RP: On a multipoint boundary value problem for a fractional order differential inclusion on an interval. Adv. Math. Phys. 2013, Article ID 823961 (2013)

17. Razminia, A, Baleanu, D, Majd, VJ: Conditional optimization problems: fractional order case. J. Optim. Theory Appl. $156,45-55$ (2013)

18. Wang, W, Huang, L: Existence of positive solution for semipositone fractional differential equations involving Riemann-Stieltjes integral conditions. Abstr. Appl. Anal. 2012, Article ID 723507 (2012)

19. Zhang, X, Liu, L: Positive solutions of superlinear semipositone singular Dirichlet boundary value problems. J. Math. Anal. Appl. 316, 525-537 (2006)

20. Webb, JRL, Infante, G: Positive solutions of nonlocal boundary value problems involving integral conditions. NoDEA Nonlinear Differ. Equ. Appl. 15, 45-67 (2008)

21. Wang, Y, Liu, L, Wu, Y: Positive solutions for a nonlocal fractional differential equation. Nonlinear Anal. 74, 3599-3605 (2011)

22. Zhang, X, Han, Y: Existence and uniqueness of positive solutions for higher order nonlocal fractional differential equations. Appl. Math. Lett. 25, 555-560 (2012)

23. Aris, R: Introduction to the Analysis of Chemical Reactors. Prentice Hall, Englewood Cliffs (1965)

24. Tao, H, Fu, M, Qian, R: Positive solutions for fractional differential equations from real estate asset securitization via new fixed point theorem. Abstr. Appl. Anal. 2012, Article ID 842358 (2012)

25. Merdan, M, Khan, T: Homotopy perturbation method for solving viral dynamical model. Fen Bilimleri Dergisi 31, 65-77 (2010)

26. Petrovic, L, Spasic, D, Atanackovic, TM: On a mathematical model of a human root dentin. Dent. Mater. 21, 125-128 (2005)

27. Tuckwell, C, Frederic, Y: On the behavior of solutions in viral dynamical models. Biosystems 73, 157-161 (2004) 
28. Perelson, A: Modeling the interaction of the immune system with HIV. In: Castillo-Chavez, C (ed.) Mathematical and Statistical Approaches to AIDS Epidemiology. Lecture Notes in Biomathematics, vol. 83, p. 350. Springer, New York (1989)

29. Yang, G: Nontrivial solution of fractional differential system involving Riemann-Stieltjes integral condition. Abstr. Appl. Anal. 2012, Article ID 719192 (2012)

30. Deimling, K: Multivalued Differential Equations. de Gruyter, Berlin (1992)

31. Lasota, A, Opial, Z: An application of the Kakutani-Ky Fan theorem in the theory of ordinary differential equations. Bull. Acad. Pol. Sci., Sér. Sci. Math. Astron. Phys. 13, 781-786 (1965)

32. Granas, A, Dugundji, J: Fixed Point Theory. Springer, New York (2005)

33. Covitz, H, Nadler, SB Jr.: Multivalued contraction mappings in generalized metric spaces. Isr. J. Math. 8, 5-11 (1970)

34. Frigon, M: Théorèmes d'existence de solutions d'inclusions différentielles. In: Granas, A, Frigon, M (eds.) Topological Methods in Differential Equations and Inclusions. NATO ASI Series C, vol. 472, pp. 51-87. Kluwer Academic, Dordrecht (1995)

35. Bressan, A, Colombo, G: Extensions and selections of maps with decomposable values. Stud. Math. 90, 69-86 (1988)

36. Castaing, C, Valadier, M: Convex Analysis and Measurable Multifunctions. Lecture Notes in Mathematics, vol. 580. Springer, Berlin (1977)

doi:10.1186/1687-1847-2013-216

Cite this article as: Ahmad and Ntouyas: Existence results for fractional differential inclusions arising from real estate asset securitization and HIV models. Advances in Difference Equations 2013 2013:216.

\section{Submit your manuscript to a SpringerOpen ${ }^{\odot}$ journal and benefit from:}

- Convenient online submission

- Rigorous peer review

Immediate publication on acceptance

Open access: articles freely available online

- High visibility within the field

- Retaining the copyright to your article 